\title{
Inflation with Planck data: A survey of some exotic inflationary models
}

\author{
Cláudio Gomes, ${ }^{1, *}$ Orfeu Bertolami, ${ }^{1, \dagger}$ and João G. Rosa ${ }^{2,+}$ \\ ${ }^{1}$ Departamento de Física e Astronomia, Faculdade de Ciências da Universidade do Porto \\ and Centro de Física do Porto Rua do Campo Alegre 687, 4169-007, Porto, Portugal \\ ${ }^{2}$ Departamento de Fśica da Universidade de Aveiro and CIDMA, Campus de Santiago, \\ 3810-183, Aveiro, Portugal
}

(Received 22 March 2018; published 29 May 2018)

\begin{abstract}
We examine some inflationary models based on modifications of gravity in the light of Planck 2015 data, such as the generalized Chaplygin inspired inflation, models based in $N=1$ supergravity and braneworld scenarios. We also show that, conversely, potentials with a very flat plateau yield a primordial spectrum similar to that of the Starobinsky model with no need to modify general relativity.
\end{abstract}

DOI: 10.1103/PhysRevD.97.104061

\section{INTRODUCTION}

Inflation is a well established paradigm in cosmology. It solves the initial conditions problems of the standard hot big bang model, namely homogeneity, isotropy, flatness of the Universe and the overabundance of magnetic monopoles putatively generated in the context of grand unified theories. It also provides a mechanism to understand the origin of the observable large scale structures due to quantum fluctuations of the inflaton field. There are many models in which this paradigm can be achieved. Therefore, over the past decades several space-based observatories have gathered cosmic information and posed severe restrictions on sets of cosmological parameters as the COBE [1], the WMAP [2] and the Planck satellites [3]. Combining cosmic microwave background data from Planck, BICEP2 and Keck Array missions with baryonic acoustic oscillation data from the SDSS-III BOSS experiment highly constrain models of inflation (see e.g., Ref. [4]).

Starobinsky's model [5] was the first proposal to solve the above problems from a modification of gravity for high curvatures, and can also be written in terms of a scalar field in the Einstein frame. This model is compatible with Planck data at $1 \sigma$ level. Afterwards, other scalar field models were proposed in the context of general relativity, as the old and new inflation scenarios [6-8].

\footnotetext{
claudio.gomes@fc.up.pt

†orfeu.bertolami@fc.up.pt

joao.rosa@ua.pt
}

Published by the American Physical Society under the terms of the Creative Commons Attribution 4.0 International license. Further distribution of this work must maintain attribution to the author(s) and the published article's title, journal citation, and DOI. Funded by SCOAP.
The first realizations of inflation are excluded on various grounds.

More recently, several inflationary scenarios were constructed in the context of alternative theories of gravity, such as $f(R)$ models (see Ref. [9] for a review). Some extensions of these theories including nonminimal inflatoncurvature couplings were also analyzed, being compatible with Planck's data for some inflationary potentials [10-13].

There are several models of cosmic inflation which are based on scalar fields from superstring and supergravity theories [14]. One of such cases is the so called D-brane inflation [15], where the inflaton is a geometric modulus corresponding to the distance between two stacks of threedimensional branes and anti-branes, with the Standard Model particles being confined to the branes that survive brane-antibrane annihilation at the end of inflation. The quadratic [16] and quartic [17,18] models are compatible with Planck's results [3].

Recently, superconformal single or multifield inflationary models have attracted a significant attention since they predict universal observational parameters, at least in leading order, of the form $n_{s}=1-2 / N$ and $r=12 / N^{2}$ [19]. These results were generalized to the so-called $\alpha$-attractor inflation, where this $\alpha$ parameter is inversely related to the curvature of the Kähler manifold. For large $\alpha$, the behavior of chaotic inflation is recovered, but in the $\alpha \rightarrow 0$ limit these models converge toward the attractor behavior of the superconformal models [20]. Related scenarios involving higher curvature terms have also been considered [21].

The so-called extranatural inflation is a 5-dimensional UV-insensitive model, in which the fifth dimension is a $S^{1}$-sphere that provides the inflaton as the zero mode of a bulk $U(1)$ gauge field. The inflaton has a potential generated by at least two light charged fermions with the same $U(1)$ charges localized in the bulk, and leads to observable parameters very close to those of the Starobinsky model [22]. 
Another possibility is assuming that inflation can be driven by the Higgs field nonminimally coupled with the scalar curvature [23]. This is compatible with data [24].

Furthermore, there are other inflationary models with more than one scalar field. An example of that is the so-called hybrid inflation $[25,26]$, where a rapid rolling, or waterfall, of a scalar field, $\sigma$, is prompted by a second scalar field, $\phi$. However, these minimal hybrid models predict a blue tilted power spectrum and are therefore ruled out. However, there are other viable models of hybrid supersymmetric inflation with nonminimal Kähler potential [27].

An alternative realization of the inflationary paradigm is warm inflation, where the inflaton is coupled to other light fields [28-31]. As the inflaton slow-rolls along the potential, it loses energy that is transferred to radiation, thus heating the Universe without the need of a reheating stage after the slow-roll period.

Aside from scalar field inflation, there are also a few models on inflation driven by vector fields [32-34]. This is hard to achieve unless higher curvature terms are taken into consideration, thus allowing for an exponential expansion of the scale factor and for attractor points [35]. However, further analysis needs to be done in order to fully compare these models with existing data.

Inflation is thus a prime arena for exploring physical theories that go beyond general relativity and the standard model of particle physics, with the above discussion being just a modest sample of the large body of literature devoted to this subject. There are, however, several nonstandard inflationary models, in particular those involving modifications of general relativity, for which observational predictions have yet to be compared with the recent Planck data, a gap that we wish to fill in with this work. In Ref. [13], we have developed a numerical code to explore inflationary scenarios for a generalized Friedmann equation $H^{2}=H^{2}(\rho)$, and we will employ this tool to explore the generalized Chaplygin gas inflationary model, as well as some inflationary models within supergravity and braneworld scenarios leading to modifications of the Friedmann equation. We will show that observations allow for significant deviations from general relativity in the context of such models.

Conversely, we also show, at the end of our discussion, that general relativity can yield observational predictions that are degenerate with some modified gravity models, considering in particular the case of plateaulike potentials that yield a primordial spectrum of fluctuations similar to that of the Starobinsky model mentioned above.

This work is organized as follows. In Sec. II, we analyse the monomial and hilltop potentials in the context of the Chaplygin inspired inflation. In Sec. III, a model of $N=1$ Supergravity inflation is examined. In Sec. IV, the monomials and hilltop inflationary potentials are studied for supergravity inflation in the context of the braneworld scenario. In Sec. V, we consider the case of $N=1$ supergravity on the brane for different potentials. In Sec. VI, we explore the predictions for nearly-flat plateaux within general relativity. Finally, we summarize our main conclusions in Sec. VII.

\section{CHAPLYGIN-INSPIRED INFLATION}

The generalized Chaplygin model unifies dark matter and dark energy adopting an exotic fluid with equation of state [36,37]:

$$
p_{G C G}=-\frac{A}{\rho_{G C G}^{\alpha}},
$$

where $p_{G C G}$ and $\rho_{G C G}$ are the pressure and the energy density of the gas, respectively, and $A$ and $\alpha$ are constants, with $0<\alpha \leq 1$. The model with $\alpha=1$ was originally discussed in Refs. [36,38]. This unified model can be built with an underlying scalar field with a suitable potential [37]. ${ }^{1}$

Such an equation of state gives rise to a fluid energy density that evolves as $\rho_{G C G}=\left(A+\rho_{m}^{1+\alpha}\right)^{\frac{1}{1+\alpha}}$, where $\rho_{m} \propto$ $a^{-3}$ behaves as the energy density of nonrelativistic matter. It has been shown in Ref. [39] that such a behavior can also be obtained from a modification of gravity, in particular from a generalized Born-Infeld action for a scalar field, yielding a Friedmann equation of the form:

$$
H^{2}=\frac{1}{3 M_{P}^{2}}\left[A+\rho_{\phi}^{1+\alpha}\right] \frac{1}{1+\alpha}
$$

where $M_{P}=(8 \pi G)^{-2}$ is the reduced Planck mass. One can therefore assume that inflaton is such a scalar field, nevertheless obeying the standard classical equation of motion:

$$
\ddot{\phi}+3 H \dot{\phi}+V^{\prime}(\phi)=0 .
$$

As examples, we will consider two main cases in the slow-roll regime: $\alpha=1$ and $\alpha=0.5$ for linear and quadratic monomials inflationary potentials. However, for the quartic and quadratic hilltop models only the $\alpha=1$ case will be analysed due to numeric limitations in the computation of the observable quantities. Monomial potentials are the ones which can be cast in the following form:

$$
V(\phi)=V_{0}\left(\frac{\phi}{M_{P}}\right)^{n}
$$

where the constant $V_{0}$ sets the scale of inflation and $n \in \mathbb{Z}^{+}$. For these potentials, the slow-roll parameters read:

\footnotetext{
${ }^{1}$ Notice that the model also admits a complex scalar field construction $[37,38]$.
} 


$$
\begin{aligned}
\epsilon_{\phi} & =\frac{n^{2}}{2 \tilde{\phi}^{2}}, \\
\eta_{\phi} & =\frac{n(n-1)}{\tilde{\phi}^{2}},
\end{aligned}
$$

where $\tilde{\phi} \equiv \phi / M_{P}$.

Hilltop potentials can be written as:

$$
V(\phi)=V_{0}\left(1-\frac{\gamma}{n} \tilde{\phi}^{n}\right)
$$

where the parameter $\gamma \in] 0,1[$. The slow-roll parameters can be expressed as:

$$
\begin{aligned}
\epsilon_{\phi} & =\frac{\gamma^{2} \tilde{\phi}^{2 n-2}}{2\left(1-\frac{\gamma}{n} \tilde{\phi}^{n}\right)^{2}}, \\
\eta_{\phi} & =-\frac{(n-1) \tilde{\phi}^{n-2}}{1-\frac{\gamma}{n} \tilde{\phi}^{n}} .
\end{aligned}
$$

The number of e-folds of inflation after horizon-crossing reads:

$$
N_{e}=-\frac{1}{M_{P}^{2}} \int_{\phi_{*}}^{\phi_{e}} \frac{\left(A+V(\phi)^{1+\alpha}\right)^{\frac{1}{1+\alpha}}}{V^{\prime}(\phi)} d \phi
$$

where the value of the inflaton at the end of inflation for a generic modified Friedmann equation of the type $H^{2}=$ $H^{2}(\rho)$, is given by the strongest of the two conditions for each potential [13]:

$$
\begin{gathered}
\epsilon_{\phi} \sim 3\left(\frac{M_{P}^{2} H^{2}}{V}\right)^{2}\left(M_{P}^{2} \frac{d H^{2}}{d \rho}\right)^{-1}, \\
\epsilon_{\phi} \sim 9 \frac{H^{2} M_{P}^{2}}{V},
\end{gathered}
$$

where the first condition signals the end of accelerated expansion and the second condition the failure of the slowroll condition $\dot{\phi}^{2} / 2<V(\phi)$. Note that these two conditions coincide in general relativity with a canonical scalar field, but are in general distinct when one considers deviations from the standard Friedmann equation, as is the case of the generalized Chaplygin inflation scenario.

The scalar spectral index and the tensor-to-scalar ratio are given by:

$$
\begin{aligned}
n_{s} & =1-6 \epsilon_{\phi_{*}} \frac{V\left(\phi_{*}\right)^{2+\alpha}}{\left(A+V\left(\phi_{*}\right)^{1+\alpha}\right)^{\frac{\alpha+2}{\alpha+1}}}+\frac{2 \eta_{\phi_{*}} V\left(\phi_{*}\right)}{\left(A+V\left(\phi_{*}\right)^{1+\alpha}\right)^{\frac{1}{1+\alpha}}}, \\
r & =16 \epsilon_{\phi_{*}} \frac{V\left(\phi_{*}\right)^{2}}{\left(A+V\left(\phi_{*}\right)^{1+\alpha}\right)^{\frac{2}{1+\alpha}}} .
\end{aligned}
$$

Let us define $x \equiv \frac{A}{V_{0}^{1+\alpha}}$. In Fig. 1, we plot the observational predictions for the linear and quadratic potentials.

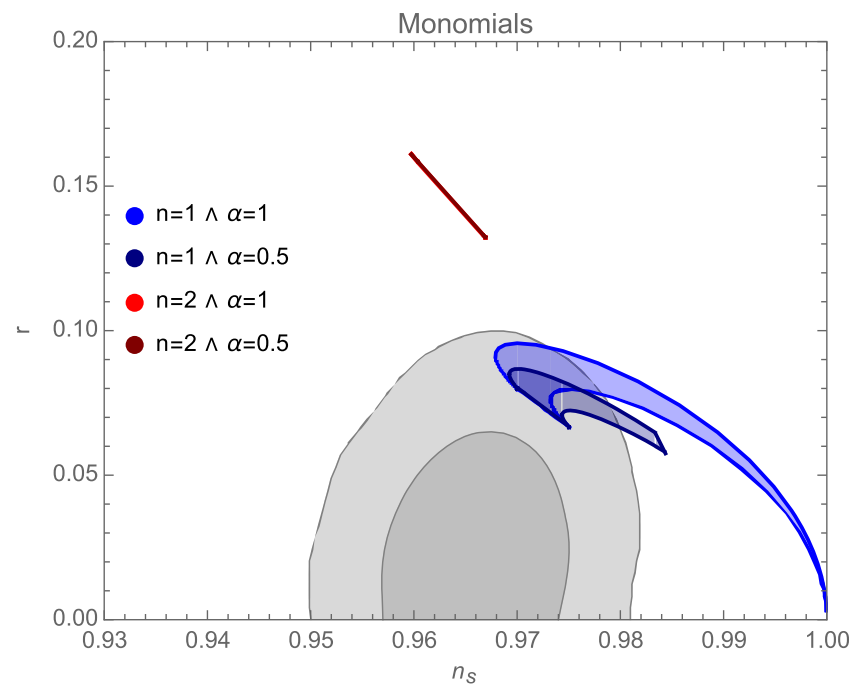

FIG. 1. Predictions for monomial potentials in the Chaplygin inspired inflation in comparison with Planck data in grey. For $n=1$, if $\alpha=0.5$, then $x \equiv \frac{A}{V_{0}^{1+\alpha}}<0.53$. For $n=2$, if $\alpha=1$, then $x<0.59$, and for $\alpha=0.5$ we must have $x<0.53$. Upper and lower bounds correspond to $N_{e}=50$ and $N_{e}=60$, respectively.

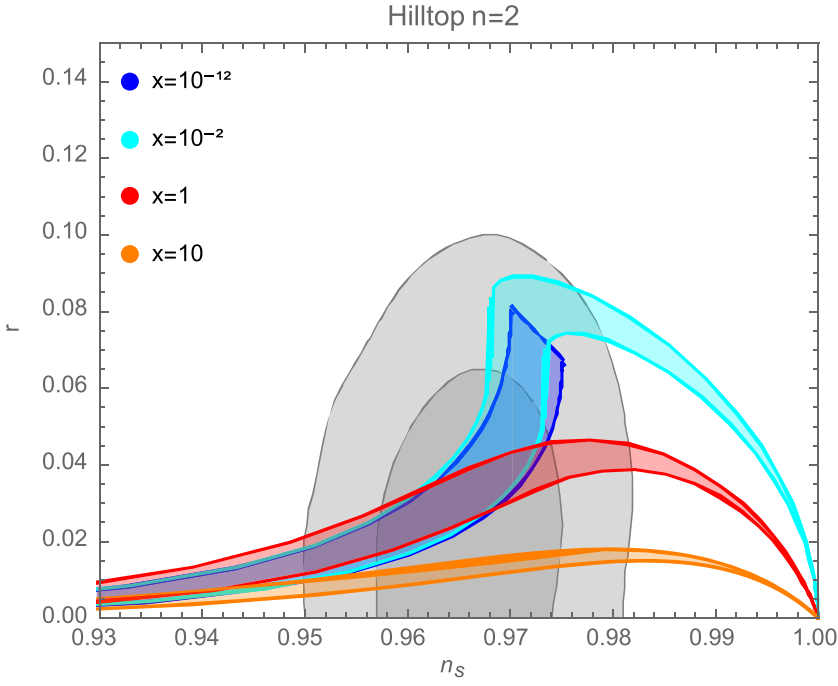

FIG. 2. Predictions for the quadratic hilltop potential in a generalized Chaplygin model with $\alpha=1$ in comparison with Planck data in grey. For $x \ll 1$, one retrieves the usual Friedmann equation prediction, whilst for larger values of $x$ the potential behavior differs from that. Upper and lower bounds correspond to $N_{e}=50$ and $N_{e}=60$, respectively.

From the condition that inflation has to end, some constraints arise for $x$ in all cases, except for the linear potential for $\alpha=1$, for which it is always possible to end inflation without further requirements on $x$ and that exhibits an attractor point at $\left(n_{s}, r\right)=(1,0)$ for $x \rightarrow \infty$.

For $\alpha=1$, the observational predictions for the quadratic and quartic hilltop potentials are shown in Figs. 2 and 3, respectively, for different values of $x$. In both cases, as $x \rightarrow 0$, 


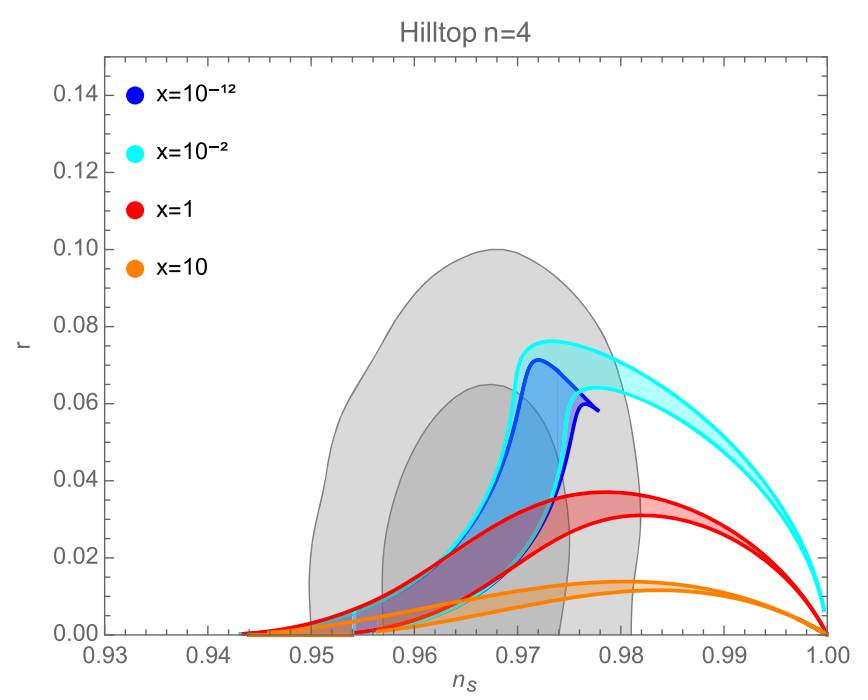

FIG. 3. Predictions for the quartic hilltop potential in a generalized Chaplygin model with $\alpha=1$ in comparison with Planck data in grey. For $x \ll 1$, one retrieves the usual Friedmann equation prediction, whilst for larger values of $x$ the potential behavior is quite different. Upper and lower bounds for each case correspond to e-folds between 50 and 60, respectively.

one retrieves the predictions of such potentials in general relativity, as expected, but for greater $x$ the deviations become significant: the tensor-to-scalar ratio becomes smaller and there is an attractor point at $\left(n_{s}, r\right)=(1,0)$ for $\gamma \rightarrow 0$. However, there is still a range of values for the free parameter that is allowed by data.

\section{III. $N=1$ SUPERGRAVITY INFLATION}

We shall now study an $N=1$ supergravity (SUGRA) potential that describes the interaction of chiral superfields, and is specified by the Kähler potential, $K\left(\Phi, \Phi^{\dagger}\right)$. The scalar potential reads [40]:

$$
V=\frac{e^{K}}{4}\left(G_{a}\left(K^{-1}\right)_{b}^{a} G^{b}-3|W|^{2}\right),
$$

where $G_{a}=K_{a} W+W_{a}$ is the Kähler function, the indices $a, b$ correspond to derivatives with respect to the chiral superfields, $\Phi$, and $W(\Phi)$ is the superpotential that describes the Yukawa and the scalar couplings of the supersymmetric theory.

We shall assume that the superpotential can be split into supersymmetry-breaking, $P$, gauge, $G$, and inflationary, $I$, sectors, such that:

$$
W=P+G+I .
$$

We will consider the supersymmetric model constructed in Refs. [41,42] with the minimal choice for the Kähler potential, $K=\Phi \Phi^{\dagger}$ and the inflaton superpotential, $I=$ $\Delta^{2} M_{P} f\left(\frac{\Phi}{M_{P}}\right)$, where $\Delta$ corresponds to the inflation scale, and $f\left(\Phi / M_{P}\right)$ is a function that is not constrained by the underlying $R$-symmetry of the model. Thus, the scalar potential in terms of the inflaton field, $\phi$, reads [40]:

$$
V_{I}(\phi)=e^{|\phi|^{2} / M_{P}^{2}}\left(\left|\frac{\partial I}{\partial \phi}+\frac{\phi^{*} I}{M_{P}^{2}}\right|^{2}-\frac{3|I|^{2}}{M_{P}^{2}}\right)_{\Phi=\phi} .
$$

Requiring that SUSY remains unbroken in the global minimum, $\left|\frac{\partial I}{\partial \Phi}+\frac{\Phi^{*} I}{M_{P}^{2}}\right|_{\Phi=\phi_{0}}=0$, and that the present cosmological constant vanishes, $V_{I}\left(\phi_{0}\right)=0$, it follows that the simplest superpotential $I$ that satisfies these conditions is

$$
I(\phi)=V_{0}\left(\phi-\phi_{0}\right)^{2},
$$

where $V_{0}=\Delta^{2} / M_{P}$. This potential is flat close to the origin for $\phi_{0}=M_{P}$, which also yields a vanishing inflaton mass at this point. Thus, in the vicinity of the origin the potential is given by the Taylor expansion [41,42]:

$V_{I}(\phi)=\Delta^{4}\left(1-4 \tilde{\phi}^{3}+\frac{13}{2} \phi^{4}-8 \tilde{\phi}^{5}+\frac{23}{3} \tilde{\phi}^{6}+\cdots\right)$.

In this model, the slow-roll parameters read:

$$
\begin{aligned}
& \epsilon_{\phi} \simeq 2\left(\frac{-6 \tilde{\phi}^{2}+13 \tilde{\phi}^{3}-20 \tilde{\phi}^{4}+23 \tilde{\phi}^{5}}{1-4 \tilde{\phi}^{3}+\frac{13}{2} \tilde{\phi}^{4}-8 \tilde{\phi}^{5}+\frac{23}{3} \tilde{\phi}^{6}}\right)^{2}, \\
& \eta_{\phi} \simeq \frac{-24 \tilde{\phi}+78 \tilde{\phi}^{2}-160 \tilde{\phi}^{3}+230 \tilde{\phi}^{4}}{1-4 \tilde{\phi}^{3}+\frac{13}{2} \tilde{\phi}^{4}-8 \tilde{\phi}^{5}+\frac{23}{3} \tilde{\phi}^{6}} .
\end{aligned}
$$

The number of e-folds follows from the integration:

$$
N_{e}=-\frac{1}{M_{P}^{2}} \int_{\phi_{*}}^{\phi_{e}} \frac{V(\phi)}{V^{\prime}(\phi)} d \phi .
$$

In such models, the equation of motion of the inflaton and the Friedmann equation are the standard ones in general relativity. Therefore, the scalar index and the tensor-to-scalar ratio are straightforwardly computed at horizon crossing:

$$
\begin{gathered}
n_{s}=1-6 \epsilon_{\phi_{*}}+2 \eta_{\phi_{*}}, \\
r=16 \epsilon_{\phi_{*}} .
\end{gathered}
$$

This model is ruled out, since it predicts a scalar index in the range $n_{s} \in[0.92,0.94]$ which is excluded by the Planck data. It also leads to very small tensor-to-scalar ratio, actually of the order of $10^{-9}-10^{-8}$. Furthermore, some small field inflationary models in minimal $N=1$ SUGRA with only one chiral superfield have been analyzed in Ref. [43], and the results showed that there is some tension 
with data. However, one should note that the Planck data favors slightly lower values of the scalar spectral index when taking into account the total neutrino mass, $M_{\nu}$, and the effective number of relativistic degrees of freedom, $N_{\text {eff }}$, as free parameters, namely in scenarios with $M_{\nu}$ larger than the minimal value allowed by neutrino oscillation data and $N_{\text {eff }}<3.046$, the latter corresponding to scenarios with a low-reheating temperature [44]. Hence, we cannot exclude a consistency of the SUGRA model with data.

Since the present SUGRA model does not meet standard observational data, we shall consider supergravity inflation on the brane in the next sections.

\section{BRANE INFLATION}

In the 5-dimensional brane scenario, matter fields are confined to a 3-brane and only gravity propagates in the fifth dimension, and the 4-dimensional Einstein equations read [45]:

$$
G_{\mu \nu}=-\Lambda g_{\mu \nu}+\frac{1}{M_{P}^{2}} T_{\mu \nu}+\frac{1}{M_{5}^{6}} S_{\mu \nu}-E_{\mu \nu},
$$

where $M_{5}=\left(8 \pi G_{5}\right)^{-2}$ is the 5-dimensional reduced Planck mass associated to the 5-dimensional gravitational constant $G_{5}, T_{\mu \nu}$ is the energy-momentum on the brane, $S_{\mu \nu}$ is a tensor with contributions quadratic in $T_{\mu \nu}$, and $E_{\mu \nu}$ is the projection of the 5-dimensional Weyl tensor on the 3-brane. We assume that the brane is described by the Robertson-Walker metric, such that the Friedmann equation reads [45-47]:

$$
H^{2}=\frac{\Lambda}{3}+\frac{\rho}{3 M_{P}^{2}}+\frac{\rho^{2}}{9 M_{5}^{6}}+\frac{\epsilon}{a^{4}},
$$

where $\epsilon$ is an integration constant and $a$ is the scale factor. Since the cosmological constant is negligible and the last term of the Friedmann equation quickly vanishes when inflation sets in, the modified Friedmann equation reads $[48,49]$ :

$$
H^{2}=\frac{\rho}{3 M_{P}^{2}}\left(1+\frac{\rho}{2 \lambda}\right)
$$

where $\lambda$ is the 3 -brane tension. ${ }^{2}$

\footnotetext{
${ }^{2}$ We note that there are other similar models as the Cardassian one [50], where the Friedmann equation has an ad hoc correction of the form $\left(1+\frac{\rho^{n}}{2 \lambda}\right)$; this model has already been constrained by several observations, and both the $\Lambda \mathrm{CDM}$ and the Cardassian models are equally favored with WMAP [51], whilst gamma ray burst data further narrow the allowed values for the exponent of such model or even a modified version of it - the modified polytropic Cardassian model [52]. Another possibility is loop quantum cosmology $[53,54]$, where the corrections to the standard Friedman equation have a minus sign, $H^{2} \sim \rho\left(1-\frac{\rho}{2 \lambda}\right)$, which is compatible with data $[55,56]$.
}

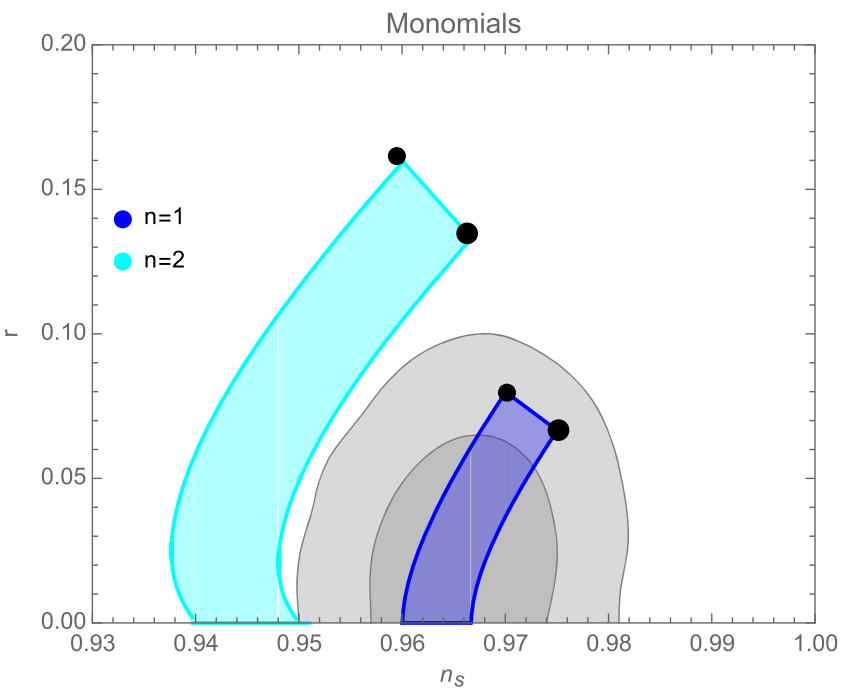

FIG. 4. Predictions for monomial potentials in a braneworld inflation scenario. For the factor $y \equiv \frac{V_{0}}{\lambda} \sim 0$, one approaches the linear or the quadratic behavior in the standard Friedmann equation, represented by the black dots, but as $y$ grows, the deviation predicts lower tensor-to-scalar ratios. The quadratic model is in conflict with Planck observations, but the linear potential is always compatible with data, and for $y \simeq 0.05$ it becomes $1 \sigma$ compatible with Planck data (in grey). Upper and lower bounds for each potential correspond to $N_{e}=50$ and $N_{e}=60$, respectively.

This model does not change the equation of motion for the fields, namely Eq. (3) (see, however, Ref. [57]).

In order to achieve a successful inflationary period, one must require the slow-roll parameters to be small. The number of e-folds of inflation follows from:

$$
N_{e}=-\int \frac{1}{\sqrt{2 \epsilon_{\tilde{\phi}}}}\left(1+\frac{1}{2 \lambda} V(\tilde{\phi})\right) d \tilde{\phi}
$$

Finally, the scalar index and the tensor-to-scalar ratio can be written as:

$$
\begin{aligned}
n_{s} & =1-6 \epsilon_{\phi_{*}} \frac{1+\frac{1}{\lambda} V\left(\phi_{*}\right)}{\left(1+\frac{1}{2 \lambda} V\left(\phi_{*}\right)\right)^{2}}+\frac{2 \eta_{\phi_{*}}}{\left(1+\frac{1}{2 \lambda} V\left(\phi_{*}\right)\right)^{2}} . \\
r & =16 \epsilon_{\phi_{*}} \frac{1}{\left(1+\frac{1}{2 \lambda} V\left(\phi_{*}\right)\right)^{2}} .
\end{aligned}
$$

For linear and quadratic monomial potentials, observational predictions are plotted in Fig. 4. The quadratic monomial model is ruled out by Planck data, but the linear model in good agreement. When the ratio $y \equiv \frac{V_{0}}{\lambda}$ grows, the tensor-to-scalar ratio gets smaller and, in particular, for the ratio $y \geq 0.05$ the linear potential becomes $1 \sigma$ compatible with data.

The quadratic and hilltop potentials are analysed in Figs. 5 and 6, respectively. As the $y$ factor grows, the 


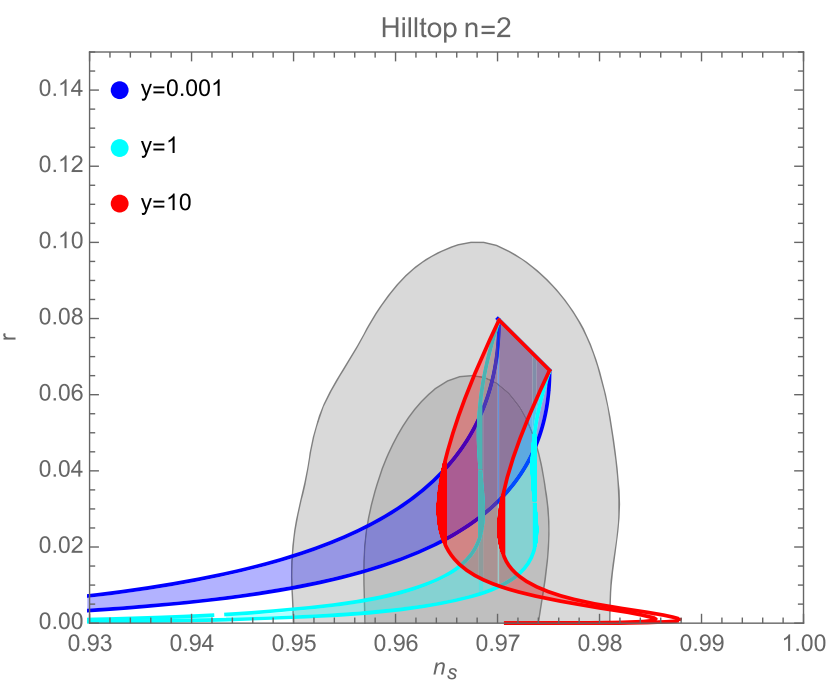

FIG. 5. Predictions for the quadratic hilltop potential in a braneworld inflationary scenario in comparison with Planck data in grey. When the factor $y \equiv \frac{V_{0}}{\lambda} \sim 0$, one gets the linear behavior with the standard Friedmann equation, but as $y$ grows, one predicts larger values of $n_{s}$ for $\gamma \rightarrow 1$. Nevertheless, they are compatible with data for a large subset of the parameter space. Upper and lower bounds correspond to $N_{e}=50$ and $N_{e}=60$, respectively.

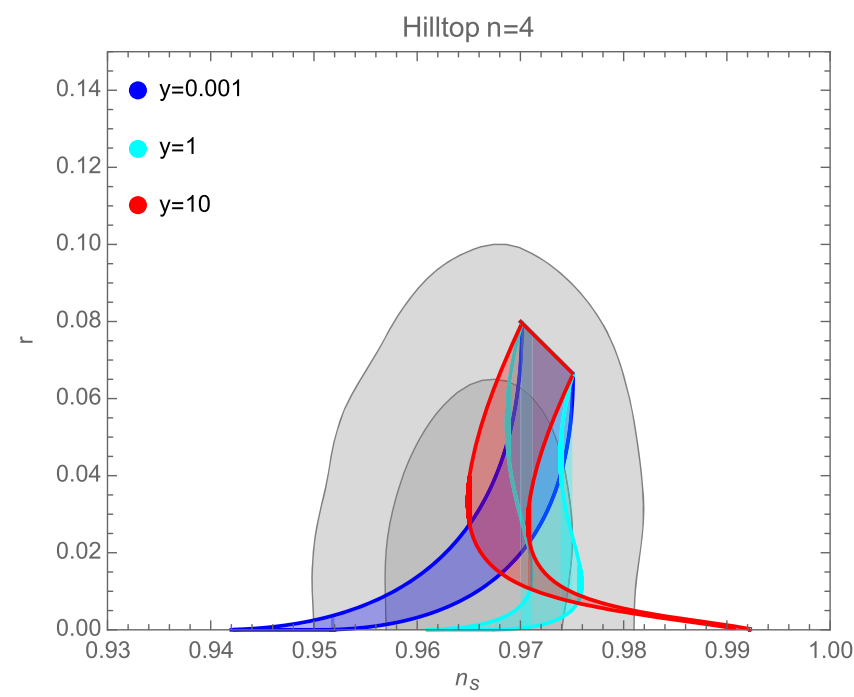

FIG. 6. Predictions for the quartic hilltop potential in a braneworld inflationary scenario in comparison with Planck data in grey. When the factor $y \equiv \frac{V_{0}}{\lambda} \sim 0$, one gets the linear potential limit with the standard Friedmann equation, but as $y$ grows, the deviation leads to larger values of $n_{s}$ for $\gamma \rightarrow 1$. Nevertheless, they are compatible with data for a large subset of the parameter space. Upper and lower bounds for each case correspond to e-folds between 50 and 60 , respectively.

predictions tend to larger values of the scalar spectral index. Notwithstanding, taking the limit $\gamma \rightarrow 0$, we recover the general relativity predictions for a linear potential, regardless of the value of the parameter $y$.

\section{V. $N=1$ SUPERGRAVITY INFLATION IN THE BRANEWORLD SCENARIO}

Let us consider the braneworld scenario discussed in the previous section, now in $N=1$ SUGRA as in Sec. III. However, instead of considering the case $\phi_{0}=M_{P}$ that leads to a hilltop-like potential, let us consider the case $\phi_{0}=0$, which yields a large-field inflationary model and consequently more significant deviations from the standard Friedmann equation.

The relevant part of the inflaton potential (along the real $\phi$ direction) then reads [49]:

$$
V(\phi)=V_{0} e^{\tilde{\phi}^{2}}\left(4 \tilde{\phi}^{2}+\tilde{\phi}^{4}+\tilde{\phi}^{6}\right),
$$

where, as before, $\tilde{\phi} \equiv \frac{\phi}{M_{P}}$. The slow-roll parameters for this potential are given by:

$$
\begin{aligned}
& \epsilon_{\phi}=2\left(\frac{4+6 \tilde{\phi}^{2}+4 \tilde{\phi}^{4}+\tilde{\phi}^{6}}{4 \tilde{\phi}+\tilde{\phi}^{3}+\tilde{\phi}^{5}}\right)^{2}, \\
& \eta_{\phi}=\frac{8+52 \tilde{\phi}^{2}+64 \tilde{\phi}^{4}+30 \tilde{\phi}^{6}+4 \tilde{\phi}^{8}}{4 \tilde{\phi}^{2}+\tilde{\phi}^{4}+\tilde{\phi}^{6}},
\end{aligned}
$$

and the number of e-folds of the inflation after horizoncrossing is computed from:

$$
N_{e}=-\int \frac{1}{\sqrt{2 \epsilon_{\tilde{\phi}}}}\left(1+\frac{1}{2 \lambda} V(\tilde{\phi})\right) d \tilde{\phi}
$$

This potential predicts a very small tensor-to-scalar ratio as shown in Fig. 7, being compatible with Planck

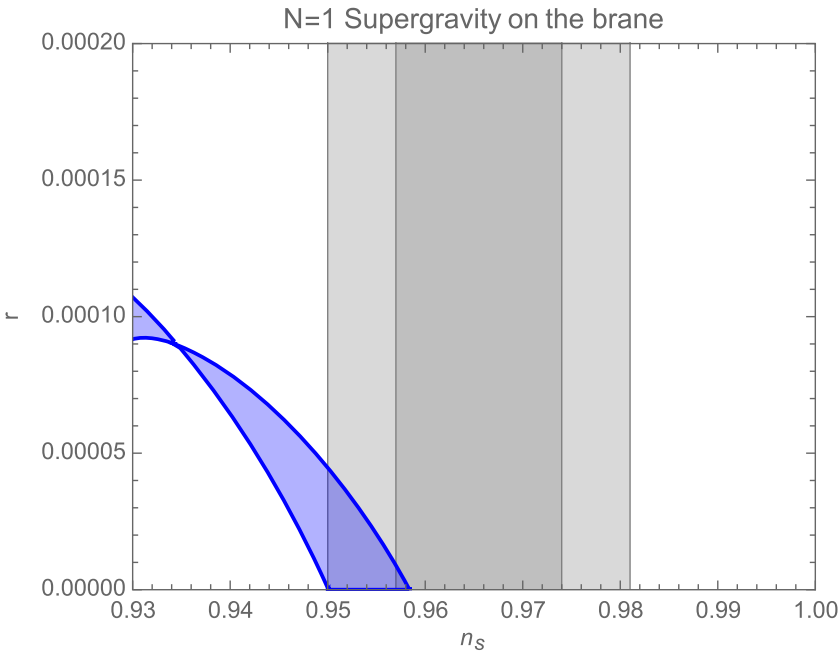

FIG. 7. Predictions for the $N=1$ supergravity inflation model on the brane. When the factor $y \equiv \frac{V_{0}}{\lambda}$ increases, predictions go from the left to the right. The upper line at $n_{s}=0.93$ corresponds to 50 e-folds of inflation, and the lower one to 60 . For 50 e-folds and $y \gtrsim 10^{6}$ and for 60 e-folds and $y \gtrsim 10^{3}$ the model becomes compatible with Planck data at 2- $\sigma$ (in light grey). 
data within the $2 \sigma$ region for $y \equiv \frac{V_{0}}{\lambda} \gtrsim 10^{6}$ for $N_{e}=50$, and $y \gtrsim 2 \times 10^{3}$ for $N_{e}=60$.

\section{PLATEAU POTENTIALS IN GENERAL RELATIVITY}

An inflationary model that has become extremely popular in the recent literature is the Starobinsky model, where a de Sitter phase is driven by an $R^{2}$ correction to the Einsten-Hilbert action. This model is related to Higgsinflation and other similar scenarios with a common feature, namely a very flat and negatively curved effective scalar potential in the Einstein frame. The first data release by the Planck collaboration made it very clear that observations favored potentials with such properties, such as hilltop or natural inflation models in addition to the ones involving modifications of the gravitational sector, at least in the context of single field cold inflation. ${ }^{3}$ To complete our discussion of inflationary scenarios, we would like to adopt a different (but complementary) perspective of showing that no modifications of general relativity are really required to explain the Planck results and that, in fact, there is a degeneracy between the predictions of the Starobinsky model and that of generic plateau-like potentials within general relativity with a scalar inflaton.

For this, we define a $p$-plateau, $p>2$, as a potential function for which $p-1$ derivatives vanish at some field value $\phi_{0}$, with $V^{(p)}\left(\phi_{0}\right)<0$. Close to the central plateau value $\phi_{0}$, the potential may thus be written in the form:

$$
V(\phi)=V_{0}\left[1-\frac{\mu}{p}\left(\frac{\phi-\phi_{0}}{M_{P}}\right)^{p}+\cdots\right],
$$

where $\mu>0$. This approach is similar to the so-called inflection-point inflation within particle physics [58-60] and within string theory [61-63]. However, the first realization has a soft SUSY breaking whose potential along the flat direction reads: $V=\frac{1}{2} m_{\phi}^{2} \phi^{2}+$ $A \cos \left(n \theta+\theta_{A}\right) \frac{\lambda_{n} \phi^{n}}{n M_{P}^{n-3}}+\lambda_{n}^{2} \frac{\phi^{2(n-1)}}{M_{P}^{(2 n-3)}}$, where $\phi$ and $\theta$ are the radial and angular coordinates of the superpotential complex scalar field, $\theta_{A}$ is the phase of the A-term, $A>0$ has mass dimension, and $\lambda \sim \mathcal{O}(1)$ is a constant. Whilst the second realization has a potential term of the form $V(\phi)=V_{0}\left(1-\mu_{1}\left(\phi+\phi_{0}\right)+\mu_{2}\left(\phi-\phi_{0}\right)^{3}\right)$, for some real parameters $\mu_{1}, \mu_{2}$.

Defining $\Delta \tilde{\phi}=\left(\phi-\phi_{0}\right) / M_{P} \ll 1$, one has for the slow-roll parameters:

$$
\begin{aligned}
\epsilon_{\phi} & \simeq \frac{\mu^{2}}{2} \Delta \tilde{\phi}^{2 p-2}, \\
\eta_{\phi} & \simeq-\mu(p-1) \Delta \tilde{\phi}^{p-2},
\end{aligned}
$$

\footnotetext{
${ }^{3}$ In warm inflation, on the other hand, monomial potentials such as $V(\phi)=\lambda \phi^{4}$ are compatible with Planck data [30,31].
}

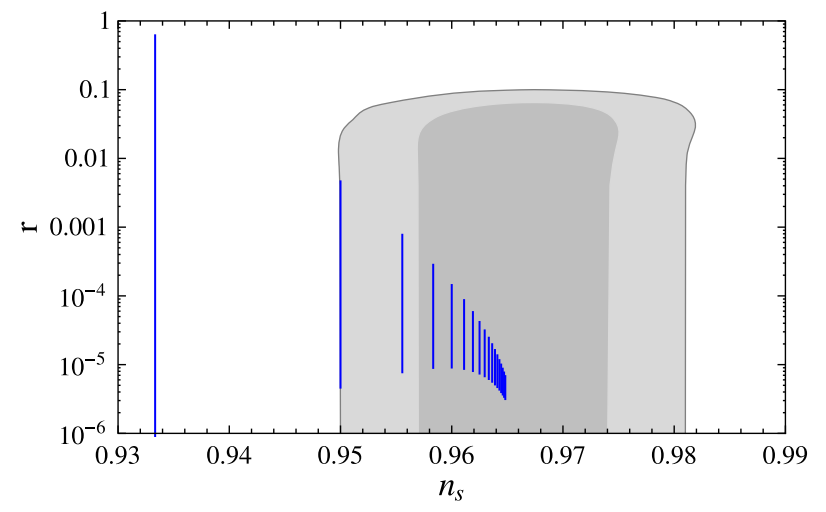

FIG. 8. Observational predictions in the $\left(n_{s}, r\right)$ plane for plateaux of order $p=3, \ldots, 20$ (increasing in unit steps from left to right), for 60 e-folds of inflation and $\mu=10^{-3}-1$. Planck data is shown in grey.

such that $\epsilon_{\phi} \ll\left|\eta_{\phi}\right|$ for $\mu \lesssim 1$. The number of e-folds of inflation after horizon-crossing of the relevant CMB scales is thus:

$$
N_{e}=-\frac{1}{M_{P}^{2}} \int_{\phi_{*}}^{\phi_{e}} \frac{V(\phi)}{V^{\prime}(\phi)} d \phi \simeq \frac{\Delta \tilde{\phi}_{*}^{2-p}}{(p-2) \mu},
$$

where we have taken into account that $\Delta \tilde{\phi}_{e} \gg \Delta \tilde{\phi}_{*}$ and $p>2$, while the spectral index and tensor-to-scalar ratio are given by:

$$
\begin{aligned}
n_{s}-1 & =-6 \epsilon_{\phi_{*}}+2 \eta_{\phi_{*}} \simeq-2 \mu(p-1) \Delta \tilde{\phi}_{*}^{p-2} \\
& \simeq-\frac{p-1}{p-2} \frac{2}{N_{e}}, \\
r & =16 \epsilon_{\phi_{*}} \simeq 8 \mu^{2} \Delta \tilde{\phi}_{*}^{2 p-2} \simeq 8 \mu^{s}(p-2)^{s-2} N_{e}^{s-2},
\end{aligned}
$$

where $s=2 /(2-p)$. For $p \gg 1$, i.e., for a very flat plateau, one then has:

$$
\begin{gathered}
n_{s} \simeq 1-\frac{2}{N_{e}}, \\
r \simeq \frac{8}{p^{2} N_{e}^{2}},
\end{gathered}
$$

where the scalar spectral index prediction coincides with that of the Starobinsky potential and Higgs-inflation with a nonminimal gravitational coupling, yielding $n_{s} \simeq$ 0.96-0.967 for 50-60 e-folds of inflation. We show in Fig. 8 the predictions for different plateaux with integer $p \geq 3$ and $\mu=10^{-3}-1$ for 60 e-folds of inflation after horizon-crossing. It is clear from this figure that observational predictions become insensitive to the curvature of the potential, $\mu$, for large $p$, and that to agree with Planck data at $95 \%$ C.L. requires a flat plateau of integer degree $p \geq 6$.

Note that the tensor-to-scalar ratio can be arbitrarily large for any finite value of $p$ by taking $\mu \rightarrow 0$, and in this sense 
such plateaux potentials are degenerate with the Starobinksy model, for which $r \simeq 12 / N_{e}^{2}$. The effective scalar potential in this model, $V(\phi)=V_{0}\left(1-e^{-\sqrt{2 / 3} \phi / M_{P}}\right)^{2}$, may in fact be seen as an infinite degree plateau at $\phi_{0} \rightarrow+\infty$ (for which the above analysis does not strictly apply). This nevertheless shows that Planck is not really pointing definitely towards a modification of gravity, but simply to any model that effectively leads to a sufficiently flat plateau in the scalar potential, within a general relativistic description.

\section{CONCLUSIONS}

Inflation is an extremely successful paradigm that solves the main problems of the hot big bang model, also providing an explanation for the origin of the cosmic structures that we observe today. There are several models that are compatible with such a mechanism, and these are very constrained or even ruled out by recent precise data obtained by the Planck mission [3].

In this work we have analyzed some examples of inflationary models that are based on different modifications of general relativity and the gravitational interactions of the scalar inflaton field.

For the inflationary model inspired by the generalized Chaplygin gas, which unifies dark matter and dark energy at late times, we have obtained agreement with the Planck data for a linear potential and for quadratic and quartic hilltop potentials, with the quadratic monomial potential being ruled out. We have also found that the departure from general relativity leads to an attractor at $\left(n_{s}, r\right)=(1,0)$ as the parameter $x=A / V_{0}^{1+\alpha}$ grows.

We have also obtained similar agreement with data for inflationary scenarios on a 3-brane for a linear potential and both the quadratic and quartic hilltop potentials, with larger deviations from general relativity leading to smaller values of the tensor-to-scalar ratio. Again the quadratic monomial model is ruled out in the brane scenario.

We have also considered an inflationary model within $N=1$ supergravity with an $R$-symmetry, yielding a hilltoplike potential with vanishing first and second derivatives at the origin, which is observationally disfavored at more than $2 \sigma$. A similar model can, however, be made compatible with the Planck data by considering a brane-localized scenario.
Finally, we have pointed out that, although we have found agreement with data for several inflationary models within modified gravity, observations do not really require going beyond general relativity, being sufficient to consider, within the context of the latter, a scalar potential with a very flat plateau section. Predictions for such models can, in fact, be degenerate with those of the popular Starobinsky model, which is essentially likewise a special plateaupotential in the Einstein frame.

Breaking this degeneracy will require not only better precision measurements of the scalar spectral index, but also of other quantities, namely those related to the primordial tensor spectrum and possibly higher-order corrections to power-law spectra such as running indices. For example, similarly to what we have obtained in Ref. [13], inflationary models with a modified Friedmann equation such as the Chaplygin-inspired model or brane inflation analysed in this work will lead to a modified consistency relation between the tensor-to-scalar ratio and the tensor spectral index, $r / n_{t}$, which can be used to assess whether modifications of the gravitational sector play an important role in the inflationary dynamics. As explored in Ref. [13], modified theories of gravity leading to a Friedmann equation of the form $H^{2}=H^{2}(\rho)$ may have a behavior that depends on the scale $\rho / M_{P}^{2} H_{0}^{2}$, where $H_{0}^{2}$ is the overall constant in the Friedmann equation. Therefore, for instance, the consistency relation between the tensor-to-scalar ratio and the tensor spectral index can differ significantly from the GR value, $r / n_{t}=-8$, for different inflationary energy scales. We hope that this work motivates further exploration of such observables and consistency relations within different models in order to better understand the type of novel physics required to successfully realize the inflationary paradigm.

\section{ACKNOWLEDGMENTS}

The work of C. G. is supported by Fundação para a Ciência e a Tecnologia (FCT) under the Grant No. SFRH/ $\mathrm{BD} / 102820 / 2014$. J.G. R. is supported by the FCT Investigator Grant No. IF/01597/2015 and partially by the H2020-MSCA-RISE-2015 Grant No. StronGrHEP690904 and by the Center for Research \& Development in Mathematics and Applications Project No. UID/MAT/ 04106/2013.
[1] N. W. Boggess, J. C. Mather et al., The COBE mission-Its design and performance two years after launch, Astrophys. J. 397, 420 (1992).

[2] E. Komatsu et al. (WMAP collaboration), Five-year Wilkinson Microwave Anisotropy Probe (WMAP) obser- vations: Cosmological interpretation, Astrophys. J. Suppl. Ser. 180, 330 (2009).

[3] P. A. R. Ade et al. (Planck Collaboration), Planck 2015 results. XX. Constraints on inflation, Astron. Astrophys. 594, A20 (2016). 
[4] Q. G. Huang, K. Wang, and S. Wang, Inflation model constraints from data released in 2015, Phys. Rev. D 93, 103516 (2016).

[5] A. A. Starobinsky, A new type of isotropic cosmological models without singularity, Phys. Lett. 91B, 99 (1980).

[6] A. H. Guth, Inflationary universe: A possible solution to the horizon and flatness problems, Phys. Rev. D 23, 347 (1981).

[7] A. D. Linde, A new inflationary universe scenario: A possible solution of the horizon, flatness, homogeneity, isotropy and primordial monopole problems, Phys. Lett. 108B, 389 (1982).

[8] A. Albrecht and P. J. Steinhardt, Cosmology for Grand Unified Theories with Radiatively Induced Symmetry Breaking, Phys. Rev. Lett. 48, 1220 (1982).

[9] A. De Felice and S. Tsujikawa, $f(R)$ theories, Living Rev. Relativity 13, 3 (2010).

[10] O. Bertolami, C. G. Boehmer, T. Harko, and F. S. N. Lobo, Extra force in $\mathrm{f}(\mathrm{R})$ modified theories of gravity, Phys. Rev. D 75, 104016 (2007).

[11] S. Nojiri, S. D. Odintsov, and P. V. Tretyakov, From inflation to dark energy in the non-minimal modified gravity, Prog. Theor. Phys. Suppl. 172, 81 (2008).

[12] D. I. Kaiser and A. T. Todhunter, Primordial perturbations from multifield inflation with nonminimal couplings, Phys. Rev. D 81, 124037 (2010).

[13] C. Gomes, J. G. Rosa, and O. Bertolami, Inflation in nonminimal matter-curvature coupling theories, J. Cosmol. Astropart. Phys. 06 (2017) 021.

[14] K. A. Olive, Inflation, Phys. Rep. 190, 307 (1990).

[15] C. P. Burgess, M. Majumdar, D. Nolte, F. Quevedo, G. Rajesh, and R. J. Zhang, The inflationary brane anti-brane universe, J. High Energy Phys. 07 (2001) 047.

[16] J. Garcia-Bellido, R. Rabadan, and F. Zamora, Inflationary Scenarios from branes at angles, J. High Energy Phys. 01 (2002) 036.

[17] S. Kachru, R. Kallosh, A. D. Linde, J. Maldacena, L. McAllister, and S.P. Trivedi, Towards inflation in string theory, J. Cosmol. Astropart. Phys. 10 (2003) 013.

[18] G. Dvali, Q. Shafi, and S. Solganik, D-brane inflation, arXiv:hep-th/0105203.

[19] R. Kallosh and A. Linde, Universality class in conformal inflation, J. Cosmol. Astropart. Phys. 07 (2013) 002; Multifield conformal cosmological attractors, J. Cosmol. Astropart. Phys. 12 (2013) 006.

[20] R. Kallosh, A. Linde, and D. Roest, Superconformal inflationary $\alpha$-attractors, J. High Energy Phys. 11 (2013) 198.

[21] S. Cecotti and R. Kallosh, Cosmological attractor models and higher curvature supergravity, J. High Energy Phys. 05 (2014) 114.

[22] M. Dhuria, G. Goswami, and J. Prasad, Extranatural inflation redux, Phys. Rev. D 96, 083529 (2017).

[23] F. L. Bezrukov and M.E. Shaposhnikov, The standard model Higgs boson as the inflaton, Phys. Lett. B 659, 703 (2008).

[24] M. Shaposhnikov, The Higgs boson and cosmology, Phil. Trans. R. Soc. A 373, 20140038 (2015).

[25] M. C. Bento, O. Bertolami, and P. M. Sá, Inflation from strings, Phys. Lett. B 262, 11 (1991); Inflation from strings II: Reheating and baryogenesis, Mod. Phys. Lett. A 07, 911 (1992).
[26] A. Linde, Hybrid inflation, Phys. Rev. D 49, 748 (1994).

[27] M. Bastero-Gil, S. F. King, and Q. Shafi, Supersymmetric hybrid inflation with non-minimal Kahler potential, Phys. Lett. B 651, 345 (2007).

[28] A. Berera and L.-Z. Fang, Thermally Induced Density Perturbations in the Inflation Era, Phys. Rev. Lett. 74, 1912 (1995).

[29] A. Berera, Warm Inflation, Phys. Rev. Lett. 75, 3218 (1995).

[30] S. Bartrum, M. Bastero-Gil, A. Berera, R. Cerezo, R. O. Ramos, and J.G. Rosa, The importance of being warm (during inflation), Phys. Lett. B 732, 116 (2014).

[31] M. Bastero-Gil, A. Berera, R. O. Ramos, and J. G. Rosa, Warm Little Inflaton, Phys. Rev. Lett. 117, 151301 (2016).

[32] L. H. Ford, Inflation driven by a vector field, Phys. Rev. D 40, 967 (1989).

[33] M. C. Bento, O. Bertolami, P. V. Moniz, J. M. Mourão, and P. M. Sá, On the cosmology of massive vector fields with SO(3) global symmetry, Classical Quantum Gravity 10, 285 (1993).

[34] A. Golovnev, V. Mukhanov, and V. Vanchurin, Vector inflation, J. Cosmol. Astropart. Phys. 06 (2008) 009.

[35] O. Bertolami, V. Bessa, and J. Páramos, Inflation with a massive vector field nonminimally coupled to gravity, Phys. Rev. D 93, 064002 (2016).

[36] A. Yu. Kamenshchik, U. Moschella, and V. Pasquier, An alternative to quintessence, Phys. Lett. B 511, 265 (2001).

[37] M. C. Bento, O. Bertolami, and A. A. Sen, Generalized Chaplygin gas, accelerated expansion and dark energymatter unification, Phys. Rev. D 66, 043507 (2002).

[38] N. Bilić, G. B. Tupper, and R. D. Viollier, Unification of dark matter and dark energy: The inhomogeneous Chaplygin gas, Phys. Lett. B 535, 17 (2002).

[39] O. Bertolami and V. Duvvuri, Chaplygin inspired inflation, Phys. Lett. B 640, 121 (2006).

[40] E. Cremmer, B. Julia, J. Scherk, S. Ferrara, L. Girardello, and P. van Nieuwenhuizen, Spontaneous symmetry breaking and Higgs effect in supergravity without cosmological constant, Nucl. Phys. B147, 105 (1979); E. Cremmer, S. Ferrara, L. Girardello, and A. Van Proeyen, Yang-Mills theories with local supersymmetry: Lagrangian, transformation laws and super-Higgs effect, Nucl. Phys. B212, 413 (1983); R. Arnowitt, A. Chamseddine, and P. Nath, Locally Supersymmetric Grand Unification, Phys. Rev. Lett. 49, 970 (1982); E. Witten and J. Bagger, Quantization of Newton's constant in certain supergravity theories, Phys. Lett. 115B, 202 (1982); H. P. Nilles, Supersymmetry, supergravity and particle physics, Phys. Rep. 110, 1 (1984).

[41] R. Holman, E. Ramond, and G. G. Ross, Supersymmetric inflationary cosmology, Phys. Lett. 137B, 343 (1984).

[42] G. G. Ross and S. Sarkar, Successful supersymmetric inflation, Nucl. Phys. B461, 597 (1996).

[43] H. Bernardo and H. Nastase, Small field inflation in $N=1$ supergravity with a single chiral superfield, J. High Energy Phys. 09 (2016) 071.

[44] M. Gerbino, K. Freese, S. Vagnozzi, M. Lattanzi, O. Mena, E. Giusarma, and S. Ho, Impact of neutrino properties on the estimation of inflationary parameters from current and future observations, Phys. Rev. D 95, 043512 (2017). 
[45] T. Shiromizu, K. Maeda, and M. Sasaki, The Einstein equations on the 3-brane world, Phys. Rev. D 62, 024012 (2000).

[46] P. Binétruy, C. Deffayet, U. Ellwanger, and D. Langlois, Brane cosmological evolution in a bulk with cosmological constant, Phys. Lett. B 477, 285 (2000).

[47] E. E. Flanagan, S. H. Tye, and I. Wasserman, Cosmological expansion in the Randall-Sundrum brane world scenario, Phys. Rev. D 62, 044039 (2000).

[48] M. C. Bento, O. Bertolami, and A. A. Sen, Supergravity inflation on the brane, Phys. Rev. D 67, 023504 (2003).

[49] M. C. Bento and O. Bertolami, $N=1$ supergravity chaotic inflation in the braneworld scenario, Phys. Rev. D 65, 063513 (2002).

[50] K. Freese and M. Lewis, Cardassian expansion: A model in which the Universe is flat, matter dominated, and accelerating, Phys. Lett. B 540, 1 (2002).

[51] A. A. Sen and S. Sen, WMAP constraints on the Cardassian model, Phys. Rev. D 68, 023513 (2003).

[52] N. Liang, P.-X. Wu, and Z.-H. Zhu, Latest cosmological constraints on Cardassian expansion models including the updated gamma-ray bursts, Res. Astron. Astrophys. 11, 1019 (2011).

[53] M. Bojowald, Loop quantum cosmology, Living Rev. Relativity 8, 11 (2005).

[54] A. Ashtekar and P. Singh, Loop quantum cosmology: A status report, Classical Quantum Gravity 28, 213001 (2011).
[55] X. Zhang and Y. Ling, Inflationary universe in loop quantum cosmology, J. Cosmol. Astropart. Phys. 08 (2007) 012.

[56] T. Zhu, A. Wang, K. Kirsten, G. Cleaver, and Q. Sheng, Universal features of quantum bounce in loop quantum cosmology, Phys. Lett. B 773, 196 (2017).

[57] O. Bertolami and C. S. Carvalho, Lorentz symmetry derived from Lorentz violation in the bulk, Phys. Rev. D 74, 084020 (2006).

[58] R. Allahverdi, J. Garcia-Bellido, K. Enqvist, and A. Mazumdar, Gauge Invariant MSSM Inflaton, Phys. Rev. Lett. 97, 191304 (2006).

[59] J. C. B. Sanchez, K. Dimopoulos, and D. H. Lyth, A-term inflation and the MSSM, J. Cosmol. Astropart. Phys. 01 (2007) 015.

[60] R. Allahverdi, K. Enqvist, J. Garcia-Bellido, A. Jokinen, and A. Mazumdar, MSSM flat direction inflation: Slow roll, stability, fine tunning and reheating, J. Cosmol. Astropart. Phys. 06 (2007) 019.

[61] D. Baumann, A. Dymarsky, I. R. Klebanov, L. McAllister, and P. J. Steinhardt, A Delicate Universe: Compactification Obstacles to D-brane Inflation, Phys. Rev. Lett. 99, 141601 (2007).

[62] D. Baumann, A. Dymarsky, I. R Klebanov, and L. McAllister, Towards an explicit model of D-brane inflation, J. Cosmol. Astropart. Phys. 01 (2008) 024.

[63] S.-M. Choi and H. M. Lee, Inflection point inflation and reheating, Eur. Phys. J. C 76, 303 (2016). 